\title{
Perception of be Cared in Childbirth at the Light of the WHO Model
}

\author{
Maria Marques ${ }^{1}$, Margarida Sim-Sim ${ }^{2, *}$ \\ ${ }^{1}$ Obstetrics Department, Hospital do Espirito Santo, Evora, Portugal \\ ${ }^{2}$ Nursing Department, University of Évora, Évora, Portugal \\ Email address: \\ m.jp3@hotmail.com (M. Marques),msimsim@uevora.pt (M. Sim-Sim) \\ ${ }^{*}$ Corresponding author
}

\section{To cite this article:}

Maria Marques, Margarida Sim-Sim. Perception of be Cared in Childbirth at the Light of the WHO Model. American Journal of Nursing Science. Vol. 6, No. 6, 2017, pp. 478-485. doi: 10.11648/j.ajns.20170606.15

Received: September 15, 2017; Accepted: October 12, 2017; Published: December 27, 2017

\begin{abstract}
Background: The World Health Organization recommends preserving the physiology of childbirth. For a normal birth, the guidelines define obstetric practices in four categories, ranging from useful and to be encouraged to harmful and to be eliminated. Objective: to describe new mothers' perception of care during childbirth in the light of the World Health Organization model. Methods: Cross-sectional study. A self-administered structured questionnaire was used for data collection. Convenience sample of 180 mothers. The average age was 30.8 years-old $(\mathrm{SD}=5.31)$. Results: On their own initiative, before leaving for the maternity, women carried out at home, some care not recommended by World Health Organization, like pubic shaving (83.7\%). Category A represents above all the lack of a birth plan $(80.6 \%)$, mobility $(71.3 \%)$, massage $/ 54.2 \%)$ and light feeding (72.6\%). Positively represented are skin-to-skin contact (77\%) and breast-feeding (75.6\%). Category B highlights intravenous fluids access $(81.6 \%)$, lithotomy position for delivery $(82.9 \%)$ and directed pushing (86.9\%). Pubic shaving is recognized by about $22 \%$ of participants. The Kristeller maneuver or fundal pressure, is widely applied in Category D (59.9\%), highlighting continuous cardiotocography $(89.2 \%)$, repeated vaginal examination carried out by various professionals $(78.5 \%)$ and episiotomy (69.2\%). Conclusion: The World Health Organization model is weakly implemented. The medicalized culture of childbirth seems to predominate in phenomena of human nature.
\end{abstract}

Keywords: Labor, Normal Birth, Nursing, Midwifery, Obstetrics

\section{Introduction}

For reasons connected with the species' bio-psycho-sociocultural evolution, childbirth is not a solitary event for humans. The figure of the midwife to help in the obstetric dilemma responds to the species-specific standard of obligate midwifery [1-3]. Midwife assistance, over time and in caring for the woman in labor has led to a reduction in the rate of illness and death in mothers and ne-born children [4]. In order to improve these indicators, international organizations create guidelines [5]. The guidelines to be applied to women value physiological phenomena. Indeed, labor experiences are singularities and gone through by each woman according to her capacities $[6,7]$. The concept of a normal birth, after a pregnancy without unexpected events of 37 to 42 weeks of gestation and regular labor progress, contemplates spontaneous birth in a cephalic vertex presentation [8]. The first issue to be clarified is the sense in which the expression normal birth is used throughout this paper. In United Kingdom, the definition of normal birth or spontaneous delivery was the object of a clinical study in recognition of the need for its local and International clarification. In this concept, there is no place for aspects that go beyond the physiological understanding of the term normal. So there is rejection of elective induction before 41 weeks, spinal analgesia and general anesthesia, use of forceps, vacuum assistance or Caesarean, routine episiotomy and continuous cardiotocography (CTG) [9].

In hospital care, the midwife, or in the Portuguese terminology, the Nurse Specializing in Maternal Health (EESMO) has the skills to care for the woman from admission and during labor. Midwife care and technicalscientific development have contributed to positive responses 
$[8,10]$. Personalized assistance and freedom of choice, supported by informed decisions, are the rights of women in childbirth [11].

In efforts over several decades, the World Health Organization (WHO) has argued for non-medicalized action in assistance for the low-risk expectant mother $[4,8,12]$. For a normal birth, the WHO guidelines [8] define obstetric practices in four categories, ranging from useful and to be encouraged to harmful and to be eliminated. They determine a model which is less intrusive by the professional and more pro-active by the woman. In this model of assistance, the physiological process and dynamics of each birth is respected and technological resources are used with caution $[4,8]$. Considering the WHO model, it will be opportune to find out the mother's perspective. The aim of this study is therefore to describe mothers' experience of assistance during labor in the light of the WHO recommendations.

\section{Methods}

\subsection{Study Design and Population}

This is a descriptive, cross-sectional study with data collected from a hospital environment and carried out in an academic context. Data collection took place in the puerperium of a hospital in Portugal in 2016.

\subsection{Sample}

The hospital has a total of 1162 births in 2016. The criteria for selecting parturient women were: a) low-risk pregnancy (i.e. Norm $n^{\circ}$ 037/2011 of the General Board of Health), b) single fetus pregnancy, c) Portuguese speakers. Women admitted for elective Caesarean/C-section, were excluded.

The sample was calculated from 1162 births registered in the referral hospital. The number of births per month $(n=97$ births) was calculated on average and multiplied by four months, since it was, in academic context, the time available for data collection. A total of 388 births were obtained. Considering the criterion of Krecje and Morgan [13], a sample was calculated between 165-190 participants and 210 participants were invited to participate. At the time, as individuals entered the puerperium, a convenience sample of 180 women who agreed to participate was obtained.

\subsection{Instrument}

The questionnaire was applied at approximately 24 hours after the birth, after signing informed consent. The questionnaire contained two sections: a) socio-demographic aspects and obstetric history, and b) a set of dichotomous variables (i.e. yes; no), that reproduce the categorization of practices to encourage (category A), eliminate (category B), avoid (category $\mathrm{C}$ ) or that are inappropriately used (category D) [8].

\subsection{Ethical Considerations}

The current study is part of an academic research.
Permission to conduct the current research was obtained from Ethics Committee of the Research Centre for Health Sciences and Technology at the University of Évora. The hospital gave permission to carry out the study, following a positive opinion from the Commission on Ethics, registered under number 785. Each informant was requested to sign the consent form before completing the questionnaire.

\subsection{Data Analysis}

Data was analyzed using the IBM SPSS $®$, version 24.0.

\section{Results}

\subsection{Sample Descriptive Statistics}

The participants were between the ages of 17 and 43 $(\mathrm{M}=30,81 ; \mathrm{SD}=5,31)$. This was the first birth for most of the mothers $(\mathrm{N}=101 ; 56.1 \%)$. For 59 participants $(32.8 \%)$, this was the second birth and for $20(11.2 \%)$ it would be the third or more. Data from the obstetric and vaginal examination on admission to the health unit are summarized in Table 1.

Table 1. Obstetric condition on the date of admission.

\begin{tabular}{lll}
\hline & $\mathbf{n}$ & \% \\
\hline Cervical dilation & 131 & 72.8 \\
$0-3$ & 24 & 13.3 \\
$4-6$ & 3 & 1.7 \\
$7-9$ & 3 & 1.7 \\
10 & 19 & 10.6 \\
Missing & & \\
State of membranes & 120 & 66.7 \\
Complete & 60 & 33.3 \\
Broken & & \\
Birthmark & 46 & 25.6 \\
Yes & 134 & 74.4 \\
No & & \\
Uterine dynamics & 1 & 48.9 \\
Present & 88 & 51.1 \\
Absent & 92 & 3.4 \\
Gestational age & & 78.9 \\
$<37$ & 6 & \\
$37-40$ & & \\
41 & & \\
Missing & & \\
\hline & & \\
\hline
\end{tabular}

Note: $\mathrm{n}=180$

\subsection{Women Self-care Before Going to Hospital}

The time between being admitted to the health unit and the birth was 13.62 hours (i.e. $13 \mathrm{~h} 37 \mathrm{~m}$ ) on average, with a minimum of 10 minutes and a maximum of 95 hours $(3,9$ days). Epidural was used by most of the participants $(n=110$; $61.5 \%)$.

On their own initiative, before leaving for the maternity, they carried out the care presented in Figure 1. 


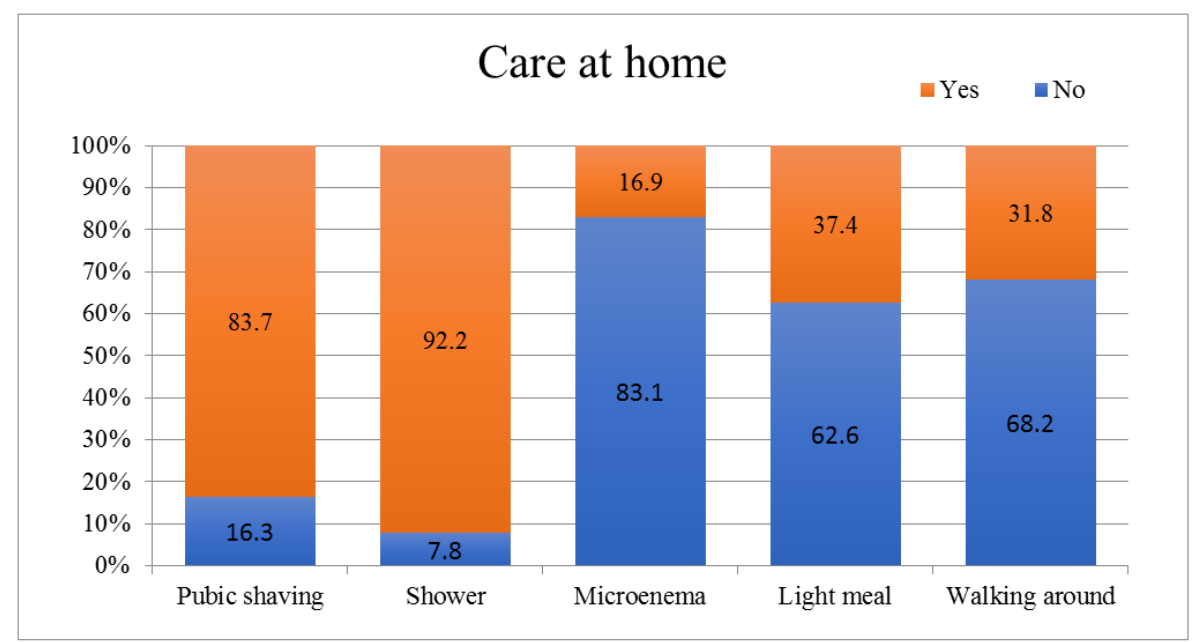

Figure 1. Care at home on own initiative.

The mothers' perception is now described, regarding the procedures they were subject to, in the light of the WHO recommendations.

\subsection{Women Perception About Nursing Care Considering Category A}

Concerning category A, the presence of the partner (97.2\% of cases) and recognition of privacy (94.4\%) is underlined. For a large percentage, the birth plan did not exist as proposed (i.e. 80.6\%) and permission to take light food was not found for $72.6 \%$. The detailed data are shown in figure 2 .

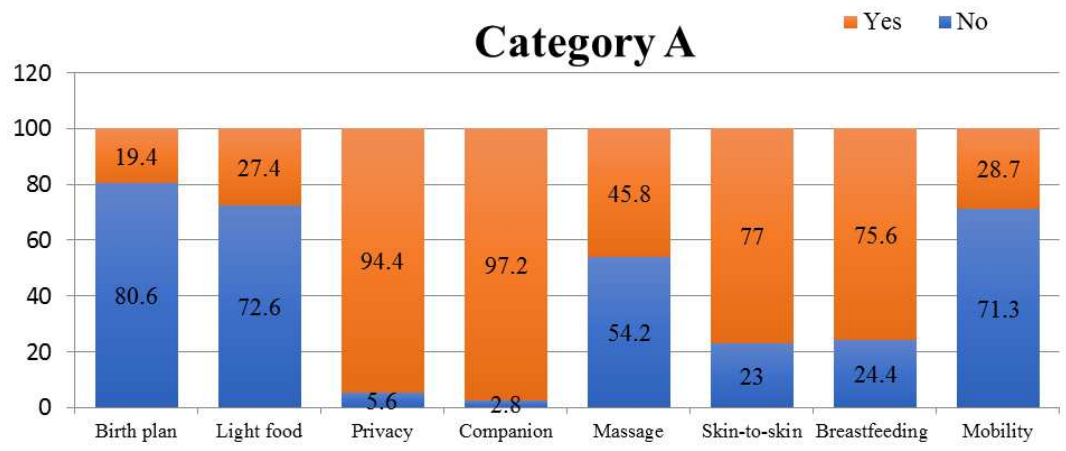

Figure 2. Category A. Useful practices to be encouraged.

\subsection{Women Perception About Nursing Care Considering Category B}

Considering category B, which includes clearly harmful practices that should be eliminated, the mothers' perception was as follows. The majority mention the EESMO's assisting practices, such as routine application of intravenous fluids $(81.6 \%)$, assistance in delivery in lithotomy $(82.9 \%)$ and directed pushing ordered by the nurse (86.9\%) (Figure 3).

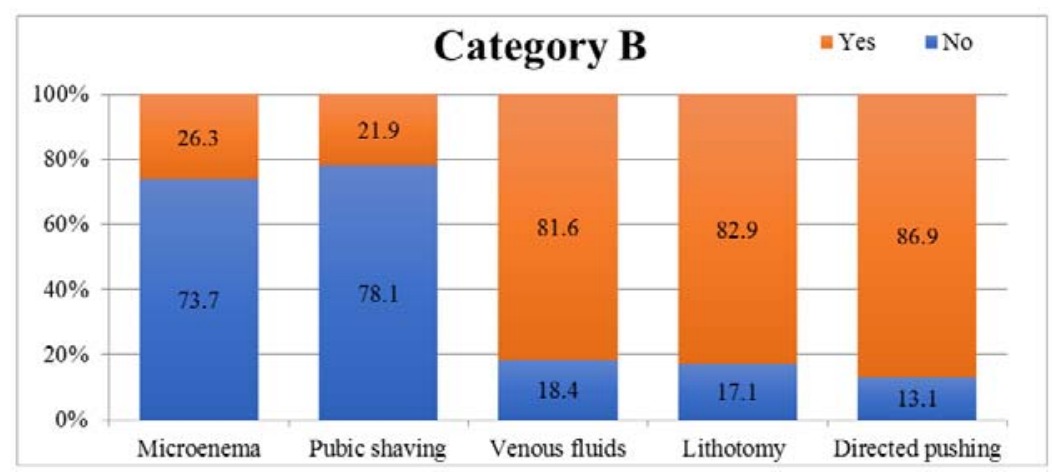

Figure 3. Category B. Clearly harmful practices to be eliminated. 


\subsection{Women Perception About Nursing Care Considering Category C}

In category $\mathrm{C}$, concerning practices where scientific evidence is insufficient to support clear recommendation and which should be used with caution, there is a high representation of the Kristeller maneuver (i.e. fundal pressure), recognized by $59.9 \%$ of mothers.

\subsection{Women Perception About Nursing Care Considering Category $D$}

Regarding category D, containing practices that are frequently used inappropriately, the results are as follows. There is greater representation of participants referring to continuous CTG $(89.2 \%)$, vaginal examination carried out by various professionals (78.5\%) and episiotomy (69.2\%) (Figure 4).

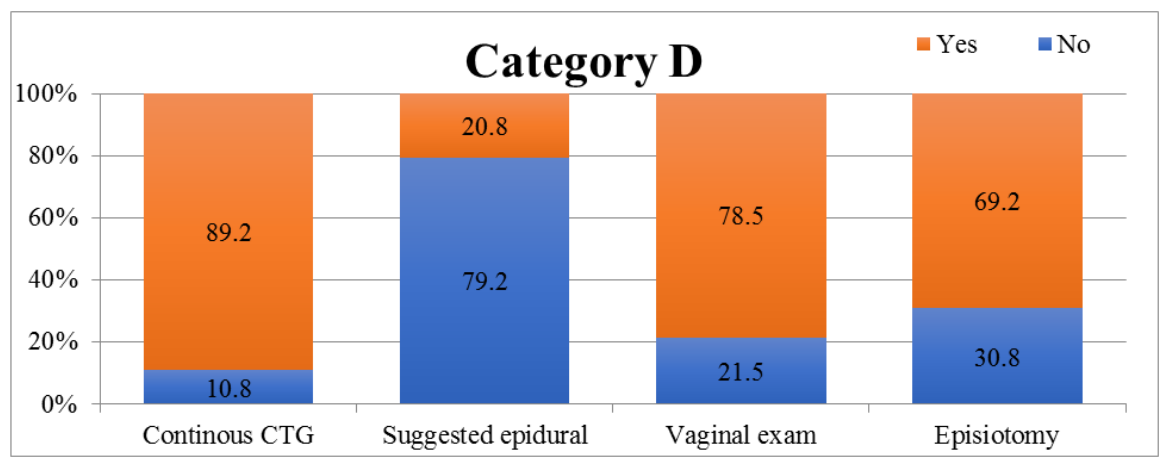

Figure 4. Category D. Practices frequently used inappropriately.

\section{Discussion}

\subsection{Discussing Women Self-care Before Going to Hospital}

Mothers are subject to practices or paradigms that predominate in the clinic, in the institutional culture regarding labor. The procedures expectant mothers carry out at home, namely pubic shaving, microenema, showering and having a light meal, reproduce a hospital care model. Therefore, the results suggest that medicalization of the physiological process is disseminated and accepted by some expectant mothers. Indeed, the medicalized view centers birth on the health professional's knowledge and expertise, on institutional norms and routine practices $[4,6]$, but more recent lines of thought tend to centre the process on the woman, on her preferences and above all on scientific evidence. Perhaps women should be informed that the physiology of labor is a unique, singular process to be respected in each case of labor.

\subsection{Discussing Women Perception About Nursing Care Considering Category $A$}

The results are then discussed in the order in which they were presented, starting with Category A practices.

The weak representation of the birth plan is evident. It may be supposed that the participants leave decisions to the professionals, symbolically excluding themselves from the labor experience. This understanding and the current results go against human nature, since there is growing belief that labor manifests itself through the nesting instinct. As a result of the hormonal environment with increased prolactin and oxytocin, preparation for birth is an attribute of the species [14]. The refusal of institutions to childbirth plans and the submission of women to the management of professionals, is contrary to the typical capacities of this moment in life. On the contrary, the hospital maternity culture facilitates the exhibition of nesting behavior [15]. Forming the plan during pregnancy and the expectant mother's visit to the hospital can contribute to more real birth plan proposals. Knowing the place and the people involved, namely the midwife/midwives gives confidence. Indeed, expectant mothers retain the ancestral species-specific traits of bonds with female figures [3]. The birth plan is an instrument that will foreseeably become universal in Portuguese hospitals due to the guidelines of the General Board of Health (i.e. National LowRisk Pregnancy Surveillance Program).

Most of participants say that their privacy was respected, aspect to cultivate in the organic of the health units, since the lack of this is disturbing and a quiet, tranquil atmosphere has a positive influence on self-control. Privacy during labor is fundamental, given the highly intimate expression of the body. Not infrequently, parturient wear reduced clothing, adopt postures, mobilize themselves, make organic noises, lose typical fluids, get excited, are restless or quiet, exhibiting manifestations, usually understood as reserved. The presence of unfamiliar people may be inhibiting and increase stress [16]. In Portugal, management of the mother private space is the responsibility of the EESMO and it is important to restrict access to the birth unit to avoid discomfort, both for the mother privacy and for quietness and concentration. The production of oxytocin during labor can even be interrupted, if stress is high [7]. It is vital to give women privacy, as they also intuitively focus to live the experience [6].

Most women in this study remained in bed during labor. This is in accordance with the traditional model of assistance, in which basic care after admission to the institution includes being given a bed, meaning a space for her to occupy [17]. 
Lying in bed does not facilitate labor [2, 14]. Mobility and movement shorten the first stage of labor by approximately one hour twenty minutes on average [18]. Changing position and movement should be encouraged as this favours accommodation of the fetus to the upper and lower channels, as well as a flexible position and thereby better presentation of the cephalic diameters to the diameters of the maternal pelvis. These are contributions to the obstetric dilemma inherited from human ancestry. It is also of note that there is less probability of a Caesarean, less use of epidural and pain management is more successful [18].

The vast majority of women did not feed during PD, in accordance with the traditional practice of banning / restraining of food, although more recently is offered ice or candy to suck and clear liquids at very small intake $\mathrm{s}[17,19]$. Food restriction is originated by the knowledge of Mendelson spread in the 1940s $[19,20]$. However, revisions of random studies conclude there are no benefits or significant threats that justify the restriction of liquids and food in low-risk women [20]. In the mother with nutritional deficiency, labor can be prolonged and more painful, besides fasting does not guarantee an empty stomach. Labor requires an effort of 295 Kcal on average [21] and current belief is that it is the woman who can best judge the need to ingest [20]. This is a way to prevent dehydration, ketosis or hyponatremia [17].

The presence of the companion, generally the husband, is mentioned by the vast majority of women, respecting the human condition and legal aspects. The presence of the partner has been part of legislation for more than 30 years in Portugal and is not accessory or to be ignored. The woman in labor has an inherent need of company [6]. This is human adaptation to evolution and is common in almost all cultures. Humans do not give birth alone, unlike non-human primates [1]. The partner is the person who has the greatest emotional link to the woman, compared to professionals who, although they may accompany her, are emotionally unknown. The potential benefits that the beginning of the link brings to the triad in terms of co-parenthood should also be considered.

Massage is referred to by less than half the women. The results suggest a lack of knowledge or unavailability of the partner or EESMO, with the benefit being lost. No added expenditure is required, which is an advantage over pharmacological methods. In a model of one-to-one care, massage as a method of relief is a valuable procedure. Massaging the expectant mother is also a naturally human initiative, as faced with the suffering woman, the companion will have the intuition to warm her, to touch and massage.

Skin-to-skin contact and breastfeeding are recognized by around $3 / 4$ of the participants. Skin-to-skin contact is defended by various authors and immediate breastfeeding becomes possible. This stabilizes the cardio-respiratory rhythm of the new-born child, reduces crying, lessens the probability of feeding with a formula, strengthens the mother-child bond and increases maternal satisfaction, besides maintaining body temperature and being a factor in reducing the child's stress $[22,23]$. Even in cases of Caesarean birth, these forms of care should be encouraged [23].

\subsection{Discussing Women Perception About Nursing Care Considering Category $B$}

Continuing the discussion now referred to as category $\mathrm{B}$, it is observed that the number of women who performed the enema is high, and the number of women carrying out microenema was high (i.e. reaching $64 ; 36 \%$ of the sample). Enema is inappropriate as preparation for a normal birth and is performed according to the health professional's preference or departmental routines. It does not support significant differences in rates of maternal death or illness in childbirth or neo-natal infection, nor in the length of labor [24]. In this study, the 64 participants were not subject to enema, but to microenema (i.e., rectal administration of 5-10 $\mathrm{ml}$ of sodium citrate and sodium lauryl sulfoacetate), with contact laxative properties, which produces freeing of the water with liquefaction of the faecal material. In fact, the literature does not reveal systematic reviews exactly about this form of microenema.

Regarding pubic shaving, most of women performed this on their own initiative at home $(n=149 ; 83.7 \%)$ and of those who did not do so at that time, it was performed by the EESMO on 12 (41.4\%). So, shaving occurred in 161 cases $(89.4 \%)$. The understanding in favour of pubic shaving defends the idea of hygienic, prevention of infection, better access to tissues in episiotomy/episiorrhaphy, or in the possible operation of instrumental birth from below [25, 26]. It is considered a routine procedure in some institutions [25]. Studies do not confirm positive results and mention discomfort through growth of pubic hair, irritation in the vulva area, multiple superficial cracks, burning sensation, heat and itching [26]. Another aspect, rarely approached, is the representation of adult femininity. Pubic hair, appearing in puberty, sexualizes the woman appearance and the pregnant body, as a result of intercourse, is expected to have a mature, adult image. Removing pubic hair makes the powerfully reproductive body more childlike, giving a distorted image of femininity.

Routine application of intravenous fluids occurs in most of expectant mothers $(81.6 \%)$. This is a frequent practice, justified by ease of access in case of emergency, or to supply fluids in case of a decision for epidural, or as a protocol of admission [25]. However, there is insufficient evidence supporting routine administration of serum [16]. Administering serum as a routine measure triggers restrictions of their movement, changes in posture, legstrengthening, exercises on all fours, use of the ball or other instruments, and leads to immobility in bed. Administration of intravenous fluids is justified for clinical reasons or due to maternal ketosis. Nevertheless, care should be taken, as this can cause breast edema and affects breastfeeding, overload liquids diminishing uterine contractility, or facilitate hyperglycemic crisis in the mother and fetus and hypoglycemia in the new-born child [27]. Most of participants $(83 \%)$ referred to lithotomy or recumbent dorsal position with stirrups in their experience during labor. In Western society, this has been the instituted position since 
childbirth moved to a hospital environment [28].

However the evidence shows the benefits of verticalized positions in mothers without epidural, although blood loss can be above $500 \mathrm{ml}$. Considering the woman's interests above all, it will be up to her to choose the most comfortable position for delivery [28].

Around $87 \%$ of the mothers state that the pushing efforts were managed by the EESMO, favouring directed pushing over spontaneous pushing. Pushing is the body's manifestation of the Ferguson reflex. It is an organic reaction, as the fetus puts pressure on the pelvic floor, communicating to the cervix that it is beginning to stretching, causing the release of endogenous oxytocin from the receptors of the vaginal tract [14]. In directed pushing, the mother is instructed to hold her breath and make perineum pushing efforts, until the end of the contraction. In spontaneous pushing, she makes pushing efforts according to the urgency she feels in her body. The results of the current study contradict the recommendations of WHO [8] and the evidence, not recognizing a major benefit in directed pushing. Women should be encouraged to act according to their preferences and the singularity of each clinical situation will guide decisions as to the type of pushing [29]. In this study, the majority of women carried out epidural, which may have contributed to high representation of directed pushing [27].

\subsection{Discussing Women Perception About Nursing Care Considering Category $C$}

Fundal pressure during delivery to facilitate exit, called the Kristeller maneuver [2], was recognized by around $60 \%$ of the women. There are potential risks for the mother, such as, uterine rupture, tearing of the anal sphincter, and situations of dyspareunia and perineal pain. Other less common risks to the mother include fractured ribs, abdominal, hematomas, liver or spleen damage, and utero-overian pedicle damage [30]. For the child the risks are cerebral damage, fractures, as well as excessive mother-child transfusion aggravated in the case of rhesus incompatibility, HIV infection, through hepatitis B or another viral disease [30, 31]. The Kristeller Maneuver has created controversy, as professionals do not agree about orientations that reject routine use [30]. In Spain there is a recommendation not to apply it (i.e. Guides for clinical practice in the NHS. 2011; p. 148). In France, guidelines say there are no validated clinical indications (i.e. Health Authority, 2007, p. 4). A Portuguese publication rejects its routine use [11]. Other authors, recommend that this maneuver should be performed by a qualified technician, warning that it is not harmless, as it may affect uterine contractility and lead to hypertonia with detrimental effects on the fetus. It may still cause the placenta to prematurely dislocate and even amniotic embolism [2].

\subsection{Women Perception About Nursing Care Considering Category $D$}

Regarding Category D, we begin the discussion with the application of the continuous CTG, it is observed that almost all the parturient recognize that it was applied (89.2\%). The results agree with that, but are higher than those found in other researches [27]. This may mean relegation of clinical knowledge, in favour of data provided by electronic equipment, or a care model with less physical presence of the EESMO. CTG is frequently performed 20 minutes after the admission, but there is no evidence of benefits for low-risk women [32]. Carrying this out on entry to maternity represents a defensive style of care, but adds $20 \%$ to the probability of a Caesarean or instrumental birth [27, 32].

The WHO recommends this should not occur with lowrisk women continuously throughout labor [8]. It does not seem to bring benefits over intermittent fetal auscultation. Telemetry is an option if there is an indication for continuous monitoring.

Epidural was suggested by EESMO in $20.8 \%$ of cases and was used by $61.5 \%(n=110)$ of the women, representation being therefore close to American studies [27]. As a means to alleviate pain, it is widely used and can be incorrectly included in the concept of a normal birth [9]. In women with epidural, the absence of pain leads to a fall in endogenous oxytocin and the need for synthetic oxytocin. This synthetic form does not cross the haemato-encephalic barrier and there is no release of endorphins. Relaxation of the pelvic muscles interferes with the fetal fall, as the cardinal movements are difficult to achieve, besides the higher risk of occiput posterior presentation [27]. Instrumental birth is therefore frequent. Indeed, pain is the woman's greatest fear and the perspective of the epidural can give confidence. Nevertheless, women should be informed that the epidural frequently leads to administration of oxytocic perfusion, motor block, a drop in blood pressure, a bad fetal position, a longer delivery, retention of liquids and greater use of forceps or vacuum [27]. Less production of endogenous oxytocin diminishes the mechanism protecting against stress in the perinatal stage and therefore affects the mother's availability, namely for breastfeeding [33].

The vaginal examination is considered next, and repeated occurrence was referred to by most participants. This is a normal procedure to determine the progress of labor, which should be used sparingly [17, 34]. There is a risk of chorioamnionitis and puerperal infection, with the observation that seven or more vaginal examination make the new-born child 4, 5 times more likely to contract infection [17]. It is an intrusive procedure that can make the woman feel physically and emotionally uncomfortable, besides confining her to bed.

Finally episiotomy, which is recognized by 117 women $(69.2 \%)$ and confirms data from other studies [27]. It is a procedure requiring the competence of the EESMO regarding the decision to perform it and the correct moment. Routine episiotomy is currently less practiced in hospitals in developed countries and is recognized in Portugal as a practice to be avoided $[11,35]$. The indication remains that it should not exceed $10 \%[8,35]$. A line of thought has emerged recently classifying routine episiotomy as obstetric violence [35]. 


\section{Conclusion}

Childbirth is a species-specific phenomenon, with particular bio-psycho-social and cultural characteristics. Given the medicalized view of childbirth, it becomes important to draw midwives' attention to users' perspectives. The study reveals poor implementation of health organization guidelines and limited pro-activity by mothers, as well as showing necessary potential modifications. It therefore contributes to respect for woman and the quality of services. Bringing this topic into the open may contribute to greater efforts and dedication in care related to the physiology of childbirth, plus an orientation towards non-pharmacological means, as recommended by the WHO. Parturient mothers do not apply their capacities to deal with labor, given the use of interventions that are removed from, or disturb the physiology of the phenomenon. If women wish to receive quality in obstetric care, it is important that they are informed by EESMO and that clinical practices are implemented based on evidence. Some measures to modify the practices of ESMES could be directed to the realization of continuous formation and the creation of working groups for the preparation of guiding documents. Protocols, guides or orientations, supported by evidence, created for institutions, can increase EESMO adhesion to practices that both ensure the safety of EESMO operations and provide parturient mothers with quality. Excellence of care facilitates the normal physiology of labor.

\section{Limitations}

The small number of participants prohibits the generalizability of the current study. As far as could be gathered from the bibliography, this is the first Portuguese research to study application of the WHO recommendations regarding care of parturient mothers. It must be considered that this is a small study, of a local nature and carried out in an academic context.

\section{Recommendations}

Given the guidelines defined by the WHO and the current scientific literature, the need for change is confirmed. It is necessary, during antenatal classes, to recommend women, to abandon the idea of trichotomy and enema at home. These are practices that have long been promoted, with the justification of better hygiene, the best healing, possible episiorrhaphy, the best application of resources in case of emergency of care (i.e. application of suction cups or forceps). The evidence rejects some of the routine practices with significant representation in this study. There are health gains if expectant mothers are informed about the benefits of non-medicalized labor. The material for classes to prepare for childbirth should prioritize contents that favour positive perspectives of a normal birth.

\section{Future Research}

A broader study with a random sample could be important.

\section{Conflict of Interests}

All the authors do not have any possible conflicts of interest.

\section{References}

[1] H. Dunsworth and L. Eccleston, "The evolution of difficult childbirth and helpless hominin infants," Annual Review of Anthropology, vol. 44, pp. 55-69, 2015.

[2] C. Montenegro and J. Rezende, Rezende. Obstetrícia Fundamental, 13th ed. Rio de Janeiro: Guanabara Koogan, 2014, p. 766.

[3] W. R. Trevathan and K. R. Rosenberg, "Caesarean section," (in eng), Evol Med Public Health, vol. 2014, no. 1, p. 164, 2014.

[4] J. Rocha and P. Novaes, "Uma reflexão após 23 anos das recomendações da Organização Mundial da Saúde para parto normal," Femina, vol. 38, no. 3, pp. 119-126, 2010.

[5] United Nations, The millenium development goals report 2015. New York: United Nations, 2015, p. 75.

[6] M.-C. Farver, "Birth Intuition," Nursing For Women's Health, vol. 19, no. 4, p. 380, 2015.

[7] M. Odent, Childbirth in the ages of plastics. London: Effra Parade, 2011.

[8] WHO, Care in normal birth: a practical guide. Geneve: Department of Reproductive Health \& Research. World Health Organization, 1996.

[9] AWHONN, "Joint Policy Statement on Normal Childbirth," Journal of Obstetrics and Gynaecology Canada, vol. 30, no. 12, pp. 1163-1165, 2008.

[10] UNFPA, U. N. P. Fund, Ed. The state of the world's midwifery 2014. State of the World's Midwifery 2014: A Universal Pathway-A Woman's Right to Health. Prague, 2014, p. 228.

[11] OE and APEO, Documento de consenso "Pelo direito ao parto normal. Uma visão partilhada". Lisboa: Ordem dos Enfermeiros, 2012.

[12] M. Amorim and L. Katz. (2012, 25th may 2016). Continuous support for women during childbirth: RHL commentary (last revised: 1 May 2012).

[13] L. Almeida and T. Freire, Metodologia da Investigação em Psicologia e Educação, 5a ed. Lisboa: Psiquilíbrios, 2017.

[14] J. Coad and M. Dunstall, Anatomy and Physiology for Midwives, 4th ed. Edinburgh: Elsevier, 2012, p. 456.

[15] D. J. Walsh, "'Nesting' and 'Matrescence' as distinctive features of a free-standing birth centre in the UK," (in eng), Midwifery, vol. 22, no. 3, pp. 228-39, Sep 2006.

[16] M. Iravani, M. Janghorbani, E. Zarean, and M. Bahrami, "An overview of systematic reviews of normal labor and delivery management," (in eng), Iran J Nurs Midwifery Res, vol. 20, no. 3, pp. 293-303, 2015 May-Jun 2015.

[17] L. Jansen, M. Gibson, B. C. Bowles, and J. Leach, "First do no harm: interventions during childbirth," (in eng), $J$ Perinat $E d u c$, vol. 22, no. 2, pp. 83-92, 2013. 
[18] A. Lawrence, L. Lewis, G. J. Hofmeyr, and C. Styles, "Maternal positions and mobility during first stage labour," (in eng), Cochrane Database Syst Rev, vol. 10, p. CD003934, 2013.

[19] N. C. Sharts-Hopko, "Oral intake during labor: a review of the evidence," (in eng), MCN Am J Matern Child Nurs, vol. 35, no. 4, pp. 197-203; quiz 204-5, 2010 Jul-Aug 2010.

[20] M. Singata, J. Tranmer, and G. M. Gyte, "Restricting oral fluid and food intake during labour," (in eng), Cochrane Database Syst Rev, no. 8, p. CD003930, 2013.

[21] C. R. Maganha e Melo and J. C. Peraçoli, "Measuring the energy spent by parturient women in fasting and in ingesting caloric replacement (Honey)," (in eng), Rev Lat Am Enfermagem, vol. 15, no. 4, pp. 612-7, 2007 Jul-Aug 2007.

[22] E. R. Moore, G. C. Anderson, N. Bergman, and T. Dowswell, "Early skin-to-skin contact for mothers and their healthy newborn infants," (in eng), Cochrane Database Syst Rev, vol. 5, p. CD003519, 2012.

[23] J. Stevens, V. Schmied, E. Burns, and H. Dahlen, "Immediate or early skin-to-skin contact after a Caesarean section: a review of the literature," Maternal \& Child Nutrition, Article vol. 10, no. 4, pp. 456-473, 2014.

[24] L. Reveiz, H. G. Gaitán, and L. G. Cuervo, "Enemas during labour," (in eng), Cochrane Database Syst Rev, no. 7, p. CD000330, 2013.

[25] R. F. Altaweli, C. McCourt, and M. Baron, "Childbirth care practices in public sector facilities in Jeddah, Saudi Arabia: a descriptive study," (in eng), Midwifery, vol. 30, no. 7, pp. 899909, Jul 2014.

[26] V. Basevi and T. Lavender, "Routine perineal shaving on admission in labour," (in eng), Cochrane Database Syst Rev, no. 11, p. CD001236, 2014.

[27] J. A. Lothian, "Healthy birth practice \#4: avoid interventions unless they are medically necessary," (in eng), J Perinat Educ, vol. 23, no. 4, pp. 198-206, 2014.
[28] J. K. Gupta, G. J. Hofmeyr, and M. Shehmar, "Position in the second stage of labour for women without epidural anaesthesia," (in eng), Cochrane Database Syst Rev, no. 5, p. CD002006, 2012.

[29] A. Lemos, M. M. Amorim, A. Dornelas de Andrade, A. I. de Souza, J. E. Cabral Filho, and J. B. Correia, "Pushing/bearing down methods for the second stage of labour," (in eng), Cochrane Database Syst Rev, no. 10, p. CD009124, 2015.

[30] A. Sartore et al., "The effects of uterine fundal pressure (Kristeller maneuver) on pelvic floor function after vaginal delivery," (in eng), Arch Gynecol Obstet, vol. 286, no. 5, pp. 1135-9, Nov 2012.

[31] E. C. Verheijen, J. H. Raven, and G. J. Hofmeyr, "Fundal pressure during the second stage of labour," (in eng), Cochrane Database Syst Rev, no. 4, p. CD006067, 2009.

[32] D. Devane, J. G. Lalor, S. Daly, W. McGuire, and V. Smith, "Cardiotocography versus intermittent auscultation of fetal heart on admission to labour ward for assessment of fetal wellbeing," (in eng), Cochrane Database Syst Rev, no. 2, p. CD005122, 2012.

[33] A. F. Bell, E. N. Erickson, and C. S. Carter, "Beyond labor: the role of natural and synthetic oxytocin in the transition to motherhood," (in eng), J Midwifery Womens Health, vol. 59, no. 1, pp. 35-42: quiz 108, 2014 Jan-Feb 2014.

[34] S. Downe, G. M. Gyte, H. G. Dahlen, and M. Singata, "Routine vaginal examinations for assessing progress of labour to improve outcomes for women and babies at term," (in eng), Cochrane Database Syst Rev, vol. 7, p. CD010088, 2013.

[35] I. Melo, L. Katz, I. Coutinho, and M. M. Amorim, "Selective episiotomy vs. implementation of a non episiotomy protocol: a randomized clinical trial," (in eng), Reprod Health, vol. 11, p. 66, 2014. 